\title{
Structured Dispersion Matrices From Division Algebra Codes for Space-Time Shift Keying
}

\author{
Rakshith Rajashekar, Student Member, IEEE, K.V.S. Hari, Senior Member, IEEE, and L. Hanzo, Fellow, IEEE
}

\begin{abstract}
We propose a novel method of constructing Dispersion Matrices (DM) for Coherent Space-Time Shift Keying (CSTSK) relying on arbitrary PSK signal sets by exploiting codes from division algebras. We show that classic codes from Cyclic Division Algebras (CDA) may be interpreted as DMs conceived for PSK signal sets. Hence various benefits of CDA codes such as their ability to achieve full diversity are inherited by CSTSK. We demonstrate that the proposed CDA based DMs are capable of achieving a lower symbol error ratio than the existing DMs generated using the capacity as their optimization objective function for both perfect and imperfect channel estimation.
\end{abstract}

Index Terms - Coding gain, diversity, space-time block code, space-time shift keying, STBCs from division algebras.

\section{INTRODUCTION}

$\mathbf{S}$ PATIAL MODULATION (SM) [1]-[7] is a novel low-complexity Multiple-Input Multiple-Output (MIMO) scheme that simply activates one out of $M$ transmit antennas for signalling. This scheme has attracted the attention of numerous researchers and led to a number of novel schemes [8]-[16].

Coherent Space-Time Shift Keying (CSTSK) [9] is capable of striking a flexible tradeoff between the attainable diversity and multiplexing gain [9], [12]. This scheme was shown to exhibit a better performance than the SM and SSK schemes, since it is capable of achieving both transmit- and receive-diversity, rather than only receive-diversity, as in SM. But, CSTSK needs multiple RF chains at the transmitter unlike SM. The information bits in this scheme are first partitioned into two sets, and then one of the sets is mapped to a point from a conventional signal set like $L$-QAM, or $L$-PSK, while the other set of bits to the index of a matrix from a set of $Q$ Dispersion Matrices (DM). Specifically, the CSTSK scheme activates one out of $Q(M \times T)$-element DMs, which is then multiplied by one of the legitimate symbols from an $L$-symbol constellation, where $T$ is the number of time-slots. This scheme offers a throughput independent of $M$, given by $R=\left(\log _{2}(Q \cdot L) / T\right)$ bpcu where bpcu in short for bits/channel use. The DMs in the existing scheme [9] are chosen by maximizing the mutual information over a large set of unityaverage-power, complex valued, Gaussian random matrices. We

Manuscript received October 10, 2012; revised February 11, 2013; accepted February 13, 2013. This work was supported by the the DST, India and by the EPSRC, U.K., under the auspices of the India-UK Advanced Technology Center (IU-ATC). The associate editor coordinating the review of this manuscript and approving it for publication was Prof. Azadeh Vosoughi.

R. Rajashekar and K. V. S. Hari are with the Department of Electrical Communication Engineering, Indian Institute of Science, Bangalore, India (e-mail: rakshithmr@ece.iisc.ernet.in; hari@ece.iisc.ernet.in).

L. Hanzo is with the School of ECS, University of Southampton, Southampton, U.K. (e-mail: 1h@ecs.soton.ac.uk).

Color versions of one or more of the figures in this paper are available online at http://ieeexplore.ieee.org.

Digital Object Identifier 10.1109/LSP.2013.2247997 refer to them as Capacity-Optimized DMs (CO-DM) which do not necessarily minimize the Symbol Error Rate (SER) [17]. The focus of this paper is to design structured DMs that attain a better bit error ratio (BER) performance than that given by the DMs of the existing scheme.

1

\section{CSTSK System AND SignAl Model}

We consider a MIMO system having $M$ transmit as well as $N$ receive antennas and a quasi-static, frequency-flat fading channel, yielding:

$$
\mathbf{Y}_{i}=\sqrt{\frac{\rho}{M}} \mathbf{H}_{i} \mathbf{X}_{i}+\mathbf{N}_{i}
$$

where $\mathbf{X}_{i} \in \mathbb{C}^{M \times T}$ is the transmitted Space-Time (ST) matrix, $\mathbf{Y}_{i} \in \mathbb{C}^{N \times T}$ is the received ST matrix, $\mathbf{H}_{i} \in \mathbb{C}^{N \times M}$ while $\mathbf{N}_{i} \in \mathbb{C}^{N \times T}$ are the channel- and noise-matrices, respectively. The entries of the channel- and noise-matrices are from a circularly symmetric complex-valued Gaussian distribution i.e., $\mathcal{C N}(0,1)$ and $\mathcal{C N}\left(0, N_{0}\right)$, respectively, where $N_{0}$ is the noise variance, $\boldsymbol{\rho}$ is the average Signal to Noise Ratio (SNR) at each receive antenna and $i$ indicates the block index in all the matrices. Throughout this paper we assume $M=T$, that is, we consider only full-diversity, minimum-delay DMs.

For the CSTSK scheme [9], we have $\mathbf{X}_{i}=\mathbf{X}_{i}^{(q, p)}=$ $s_{i, q} \mathbf{A}_{i, p}$, where $s_{i, q} \in \mathbb{C}$ is a symbol from an $L$-symbol constellation, $S, \mathbf{A}_{i, p} \in \mathbb{C}^{M \times T}$ is a DM from $\mathcal{D}$, a set of DMs with $|\mathcal{D}|=Q$, and $\mathbf{X}_{i}^{(q, p)} \in \mathcal{C}$, where $\mathcal{C}$ is a set of transmitted ST matrices. We note that all the DMs $\mathbf{A}_{i, p}$ satisfy the unity average transmission power constraint, i.e.,

$$
\operatorname{tr}\left[\mathbf{A}_{i, p}{ }^{H} \mathbf{A}_{i, p}\right]=T \text { for } 1 \leq p \leq Q .
$$

The notational representation of a typical CSTSK scheme used is formulated as ' $\operatorname{CSTSK}(M, N, T, Q), L$-symbol constellation' [9].

Let $\zeta_{p}$ be a product-map over a set of ordered pairs, $X=$ $\left\{\left(x_{1}, x_{2}\right) \mid x_{1} \in X_{1}, x_{2} \in X_{2}\right\}$ where $X_{1}$ and $X_{2}$ are two arbitrary sets, given by $\zeta_{p}:\left(x_{1}, x_{2}\right) \mapsto x_{1} x_{2}$. Then, the STSK mapping of a symbol is carried out by applying a DM to the transmitted ST matrix, which is formulated as $\zeta_{p}: S \times \mathcal{D} \mapsto \mathcal{C}$.

\footnotetext{
${ }^{1}$ Notations: Boldface uppercase letters represent matrices and are indexed as $\mathbf{X}_{i}$. Furthermore, $\operatorname{tr}[\mathbf{X}]$ and $\mathbf{X}^{H}$ denote the Trace and Hermitian of the matrix $\mathbf{X}$, respectively. $\mathbf{I}_{r}$ denotes an $(r \times r)$-element identity matrix. Greek letters like $\zeta$ indicate functions or mappings. Polynomials are represented as a function of $x$, for example $p(x)$. Calligraphic uppercase letters represent sets of matrices, for example $\mathcal{E} . \mathcal{D} \subset \mathcal{E}$ implies that $\mathcal{D}$ is a subset of $\mathcal{E}$ and $|\mathcal{D}|$ represents the cardinality of $\mathcal{D}$. Blackboard-bold font letters like $\mathbb{Q}$ represent fields. Upper case letters are used to represent sets, fields, and extended fields. The extended field $F=\mathbb{Q}(S)$ represents an extension of the field of rational numbers $\mathbb{Q}$ over some set $S . f_{i}$ or $f_{i, j}$ represent elements from a field or from a finite set.
} 
This mapping has to be one-to-one for the unambiguous detection of the transmitted ST matrices. Furthermore, it is desirable to have $\operatorname{rank}\left(\mathbf{X}_{i}^{(q, p)}-\mathbf{X}_{i}^{\left(q^{\prime}, p^{\prime}\right)}\right)=M$ for all $p \neq p^{\prime}$, or $q \neq$ $q^{\prime}$ in order to achieve both full-diversity, and simultaneously a high coding gain $G=\min _{\mathbf{X}_{i}^{(q, p)} \neq \mathbf{X}_{i}^{\left(q^{\prime}, p^{\prime}\right)} \in \mathcal{C}}\left|\operatorname{det} \Delta \Delta^{H}\right|^{1 / M}$, where $\Delta=\left(\mathbf{X}_{i}^{(q, p)}-\mathbf{X}_{i}^{\left(q^{\prime}, p^{\prime}\right)}\right)$, for the sake of improving the BER performance.

Upon vectorizing (1), we arrive at:

$$
\overline{\mathbf{Y}}_{i}=\sqrt{\frac{\rho}{M}} \overline{\mathbf{H}}_{i} \chi \mathbf{K}_{i}+\overline{\mathbf{N}}_{i}
$$

where, $\overline{\mathbf{Y}}_{i}=\operatorname{vec}\left(\mathbf{Y}_{i}\right) \in \mathbb{C}^{N T \times 1}, \overline{\mathbf{H}}_{i}=\mathbf{I}_{T} \otimes \mathbf{H}_{i} \in \mathbb{C}^{N T \times M T}$, $\overline{\mathbf{V}}_{i}=\operatorname{vec}\left(\mathbf{V}_{i}\right) \in \mathbb{C}^{N T \times 1}, \boldsymbol{\chi}=\left[\operatorname{vec}\left(\mathbf{A}_{1}\right), \ldots, \operatorname{vec}\left(\mathbf{A}_{Q}\right)\right] \in$ $\mathbb{C}^{M T \times Q}$, and $\mathbf{K}_{i}=[\underbrace{0, \ldots, 0}_{p-1}, s_{i, q}, \underbrace{0, \ldots, 0}_{Q-p}] \in \mathbb{C}^{Q \times 1}$. The equivalent system model of (3) is free from Inter-Channel Interference (ICI), hence facilitates both single-antenna based low-complexity Maximum Likelihood (ML) detection [16] and reduced search-complexity Matched Filtering (MF)-based near-ML detection [18].

\section{A. Connection Between STBCs and STSK Schemes}

Proposition 1: Any STBC, $\mathcal{C}$, over a signal set $S^{\prime}$ constitutes an ICI-free system, if there exists a set of matrices $\mathcal{E}$ such that the map $\zeta_{p}: S \times \mathcal{E} \mapsto \mathcal{C}$ is a bijection, where $S$ is any conventional signal set.

Proof: If there exists a set of $(M \times T)$-element matrices $\mathcal{E}$ such that the mapping $\zeta_{p}: S \times \mathcal{E} \mapsto \mathcal{C}$ is a bijection, then we have $\zeta_{p}^{-1}\left(\mathbf{X}_{k}\right) \neq \zeta_{p}^{-1}\left(\mathbf{X}_{l}\right)$ for all $\mathbf{X}_{k} \neq \mathbf{X}_{l} \in \mathcal{C}$, which implies $\left(s_{i}, \mathbf{E}_{j}\right) \neq\left(s_{i^{\prime}}, \mathbf{E}_{j^{\prime}}\right)$, where $\mathbf{E}_{j}, \mathbf{E}_{j^{\prime}} \in \mathcal{E}$. This suggests that we have either $i \neq i^{\prime}$ or $j \neq j$, or both, thus giving us $|\mathcal{E}|=|\mathcal{C}| /|S|$. Since we have $Q=|\mathcal{E}|$, and $\chi=$ $\left[\operatorname{vec}\left(\mathbf{E}_{1}\right), \ldots, \operatorname{vec}\left(\mathbf{E}_{Q}\right)\right]$, it is clear from (3) that the STBC is an ICI-free system.

We term this class of STBCs as Decomposable Dispersion Codes $(D D C)$. Note that DDCs are different from orthogonal codes whose weight matrices satisfy Hurwitz-Radon orthogonality condition.

\section{Proposed Full-Rate CDA Code BAsed DECOMPOSABLE DisPersion CODES}

In this section we show that the codes from Cyclic Division Algebra (CDA) [19] are DDCs and hence they may be used for STSK schemes. We propose a method for obtaining DMs from
CDA codes for achieving a desired rate and present a construction example for the $\operatorname{CSTSK}(2,2,2,8)$, BPSK system.

Considering codes constructed from CDAs over the field $F=$ $\mathbb{Q}\left(S, t, \omega_{M}\right)$, we get the full-diversity, full-rate $(M \times M)$-element Space-Time (ST) codes [19] given by (4), shown at the bottom of the page, where $\sigma$ is the Galois group generator that fixes $f_{i, j}$ and maps $t_{M}$ to $\omega_{M} t_{M}$, while the transcendental elements $t$ and $\delta$ are chosen from the unit circle to avoid information loss [19]. Furthermore, we have $|\mathcal{C}|=L^{M^{2}}$ as $\mathcal{C}$ is a full-rate code.

For ease of presentation, we adopt the following notation for describing the set in (4):

$$
\mathcal{C}=\left[\begin{array}{cccc}
\hat{K}^{(0,0)} & \delta \hat{K}^{(M-1,1)} & \cdots & \delta \hat{K}^{(1, M-1)} \\
\hat{K}^{(1,0)} & \hat{K}^{(0,1)} & \cdots & \delta \hat{K}^{(2, M-1)} \\
\vdots & \vdots & \ddots & \vdots \\
\hat{K}^{(M-1,0)} & \hat{K}^{(M-2,1)} & \cdots & \hat{K}^{(0, M-1)}
\end{array}\right],
$$

$\hat{K}^{(j, k)}=\left\{\sum_{i=0}^{M-1} f_{j, i}\left(\omega_{M}{ }^{k} t_{M}\right)^{i} \mid f_{j, i} \in S\right.$ for $\left.0 \leq i, j \leq M-1\right\}$.

In $\hat{K}^{(j, k)}$ of (5), the superscript $j$ captures the $M$ distinct sets containing $M$ independent symbols each, i.e., $\left\{f_{j, i}\right\}_{i=0}^{M-1}$, and the superscript $k$ is the distinct index of the coefficients of $\left\{f_{j, i}\right\}_{i=0}^{M-1}$ associated with each column in (4).

Proposition 2: A CDA code constructed over an arbitrary PSK signal set results in an ICI-free system, which hence may be viewed as a STSK scheme. Thus, the CDA codes enjoy the low-complexity detection benefits of the STSK scheme [16], [18].

Proof: We present the proof in two steps.

Step I: Let $F$ be an algebraic number field defined by $\mathbb{Q}\left(S, t, \omega_{M}\right)$, where $t$ is a transcendental element over $\mathbb{Q}(S)$, and $\omega_{M}=e^{j(2 \pi / M)}$. Let $K$ be an $M$ th degree algebraic extension of $F$ over $t_{M}=t^{1 / M}$, i.e., $K=F\left(t^{1 / M}\right)$. Thus, we can write $K=\left\{\sum_{i=0}^{M-1} f_{i}\left(t_{M}\right)^{i} \mid f_{i} \in F\right.$ for $\left.i=0,1, \ldots, M-1\right\}$.

Theorem 1: Let $S, F$ and $K$ be defined as above. Let $\zeta_{p}$ be a product mapping as defined earlier. If $F=S$ in $K$, then the $\operatorname{map} \zeta_{p}: S \times K_{l} \mapsto K$, where we have $K_{l}=\left\{\left(t_{M}\right)^{l}+\right.$ $\left.\sum_{i=0, i \neq l}^{M-1} f_{i}^{\prime}\left(t_{M}\right)^{i} \mid f_{i}^{\prime} \in S, i=0,1, \ldots, M-1\right\}$ for any $0 \leq$ $l \leq M-1$, is a bijection.

Proof: Proof is omitted here owing to space economy, but it is publically available at [20].

Applying Theorem 1 to the set along the main diagonal of $\mathcal{C}$ in (5), we arrive at

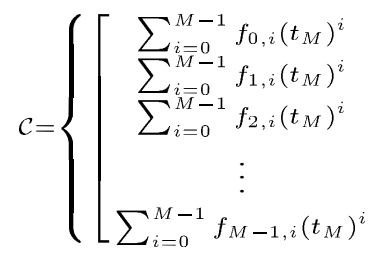$$
\begin{gathered}
\delta \sum_{i=0}^{M-1} f_{M-1, i}\left(\omega_{M} t_{M}\right)^{i} \\
\sum_{i=1}^{M-1} f_{0, i}\left(\omega_{M} t_{M}\right)^{i} \\
\sum_{i=0}^{M-1} f_{1, i}\left(\omega_{M} t_{M}\right)^{i} \\
\vdots \\
\sum_{i=0}^{M-1} f_{M-2, i}\left(\omega_{M} t_{M}\right)^{i}
\end{gathered}
$$

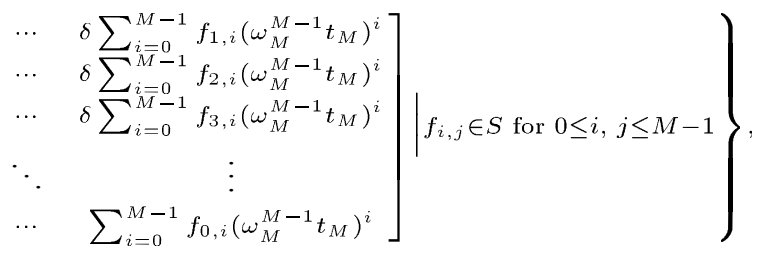


$\mathcal{C}=\left[\begin{array}{cccc}\zeta_{p}\left(S \times \hat{K}_{l_{1}}^{(0,0)}\right) & \delta \hat{K}^{(M-1,1)} & \cdots & \delta \hat{K}^{(1, M-1)} \\ \hat{K}^{(1,0)} & \zeta_{p}\left(S \times \hat{K}_{l_{2}}^{(0,1)}\right) & \cdots & \delta \hat{K}^{(2, M-1)} \\ \vdots & \vdots & \ddots & \vdots \\ \hat{K}^{(M-1,0)} & \hat{K}^{(M-2,1)} & \cdots & \zeta_{p}\left(S \times \hat{K}_{l_{M}}^{(0, M-1)}\right)\end{array}\right]$,

where, $\quad$ we $\quad \hat{K}_{l_{m}}^{(j, k)}=\left\{\left(\omega_{M}{ }^{k} t_{M}\right)^{l_{m}}+\right.$ $\sum_{i=0, i \neq l_{m}}^{M-1} f_{j, i}\left(\omega_{M}{ }^{k} t_{M}\right)^{i} \mid f_{j, i} \in S$ for $\left.0 \leq i, j \leq M-1\right\}$ for $0 \leq\left\{l_{m}\right\}_{m=1}^{M} \leq M-1$.

Step II: With the aid of Theorem 2 we will show below that the off-diagonal sets in (5) can be decomposed into the product of two sets.

Theorem 2: Let $S, K, \zeta_{p}$, and $F$ be defined as before. If we have $F=S$ in $K$, then $\zeta_{p}: S \times K \mapsto K$ is a bijection.

Proof: Proof is omitted here due to space constraints, but again, it is available at [20].

Applying Theorem 2 to the off-diagonal elements of (6), we arrive at (7), shown at the bottom of the page. Thus, from (7) we generate the bijective mapping $\zeta_{p}: S \times \mathcal{E} \mapsto \mathcal{C}$, where,

$$
\mathcal{E}=\left[\begin{array}{cccc}
\hat{K}_{l_{1}}^{(0,0)} & \delta \hat{K}^{(M-1,1)} & \cdots & \delta \hat{K}^{(1, M-1)} \\
\hat{K}^{(1,0)} & \hat{K}_{l_{2}}^{(0,1)} & \cdots & \delta \hat{K}^{(2, M-1)} \\
\vdots & \vdots & \ddots & \vdots \\
\hat{K}^{(M-1,0)} & \hat{K}^{(M-2,1)} & \cdots & \hat{K}_{l_{M}}^{(0, M-1)}
\end{array}\right]
$$

and hence, from Proposition 1 we conclude that the CDA codes result in an ICI-free system.

From (7), we can infer that $|\mathcal{C}|=L^{M^{2}}=|S| \cdot|\mathcal{E}|=L$. $L^{M^{2}-1}$ and any set $\mathcal{D} \subseteq \mathcal{E}$ can be used as a set of DMs. Thus, CDA codes offer $Q$ number of DMs, where we have $1 \leq Q \leq$ $L^{M^{2}-1}$. By contrast, for field-extension code based DMs we have $1 \leq Q \leq L^{M-1}$. We refer to these DMs obtained from the $\mathrm{CDA}$ codes as Cyclic Division Algebra code based DMs (CDA-DM). For details about CDA based DMs for QAM signal sets, refer to Section $\mathrm{V}$ in [20].

1) Example 1: Let $S=\{1,-1\}$, and $t$ as well as $\delta$ be chosen from within the unit circle. Let furthermore the number of transmit antennas be $M=2$, and $l_{1}=l_{2}=1$. From (8), we get $\mathcal{E}=\left\{\left[\begin{array}{cc}1+f_{0,1} t_{2} & \delta\left(f_{1,0}-f_{1,1} t_{2}\right) \\ f_{1,0}+f_{1,1} t_{2} & \left(1-f_{0,1} t_{2}\right)\end{array}\right] \mid f_{i, j} \in S\right\}$ with $|\mathcal{E}|=|S|^{3}=8$. In order to satisfy the unit average transmission energy constraint of (2), the matrices in the set $\mathcal{E}$ are scaled by $1 / 2$ (in general $1 / M$ ) to arrive at $\mathcal{D}=\left\{1 / 2\left[\begin{array}{cc}1+f_{0,1} t_{2} & \delta\left(f_{1,0}-f_{1,1} t_{2}\right) \\ f_{1,0}+f_{1,1} t_{2} & \left(1-f_{0,1} t_{2}\right)\end{array}\right] \mid f_{i, j} \in S\right\}$ with $Q=8$. (a)
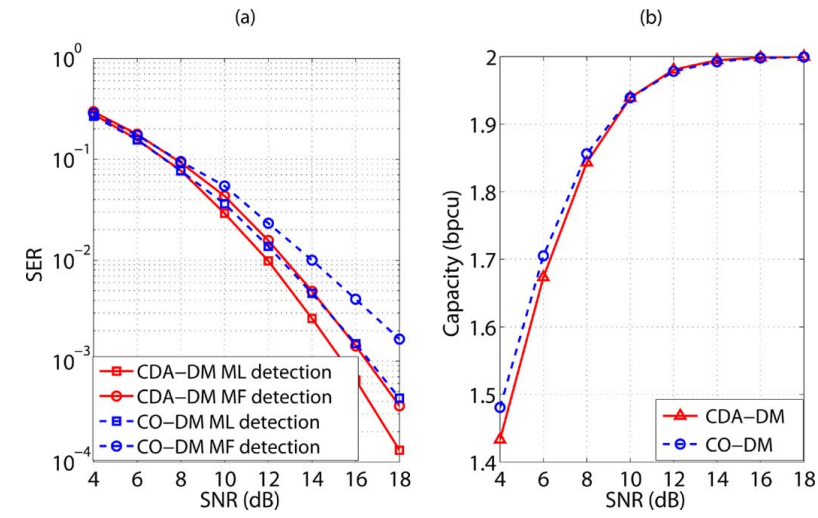

Fig. 1. SER performance and DCMC capacity curves of CDA-DM and CO-DM in CSTSK $(2,2,2,8)$, BPSK $(R=2)$. (a) ML and MF based detection in $\operatorname{CSTSK}(2,2,2,8)$, BPSK. (b) DCMC capacity of $\operatorname{CSTSK}(2,2,2,8)$, BPSK.

\section{Simulation Results and Discussion}

Simulation Scenario: In all our simulations we have used at least $10^{t+1}$ symbols, at an SER of $10^{-t}$ for evaluating the SER and assumed a block Rayleigh fading channel. The DMs of Example 1 are used for CDA-DMs with $t_{2}=e^{j(\pi / 2)}$ and $\delta=e^{j(3 \pi / 8)}$ in our simulations. CO-DMs are generated by maximizing the mutual information over a large set of DMs having complex Gaussian entries satisfying the unity-average power constraint. The coding gain offered by the CDA-DMs is one, whereas that offered by CO-DMs is 0.0455 . Hence, the proposed DMs can be expected to give a better SER performance at high SNRs than the CO-DMs. Under perfect CSIR conditions the performance of the proposed CDA-DMs, and of the CO-DMs is evaluated by considering both the ML and MF based detector of [18]. By contrast, for imperfect CSIR the iterative detection/estimation algorithm of [21] is used with 2 training and 100 data carrying STSK blocks and the performance of the Alamouti code employing 4-QAM signal set is provided for benchmarking.

\section{A. With Perfect CSIR}

Fig. 1(a) characterizes the SER performance of the proposed CDA-DM as well as of the existing CO-DM scheme in conjunction with the BPSK constellation for the $Q=8$ case. Due to the better coding gain of the CDA-DM, the proposed scheme outperforms the CO-DM scheme both for the ML and for the MF based detectors. We observe that the CDA-DM scheme exhibits an SNR gain of about $1 \mathrm{~dB}$ over the existing scheme at an SER of $10^{-3}$.

Fig. 1(b) shows the Discrete-input Continuous-output Memoryless Channel (DCMC) capacity curves of the CSTSK(2,2,2,8), BPSK scheme. We observe from Fig. 1(b) that the CDA-DM scheme approaches the capacity of the

$$
\mathcal{C}=\left[\begin{array}{cccc}
\zeta_{p}\left(S \times \hat{K}_{l_{1}}^{(0,0)}\right) & \zeta_{p}\left(S \times \delta \hat{K}^{(M-1,1)}\right) & \cdots & \zeta_{p}\left(S \times \delta \hat{K}^{(1, M-1)}\right) \\
\zeta_{p}\left(S \times \hat{K}^{(1,0)}\right) & \zeta_{p}\left(S \times \hat{K}_{l_{2}}^{(0,1)}\right) & \cdots & \zeta_{p}\left(S \times \delta \hat{K}^{(2, M-1)}\right) \\
\vdots & \vdots & \ddots & \vdots \\
\zeta_{p}\left(S \times \hat{K}^{(M-1,0}\right) & \zeta_{p}\left(S \times \hat{K}^{(M-2,1)}\right) & \cdots & \zeta_{p}\left(S \times \hat{K}_{l_{M}}^{(0, M-1)}\right)
\end{array}\right]
$$


(a)

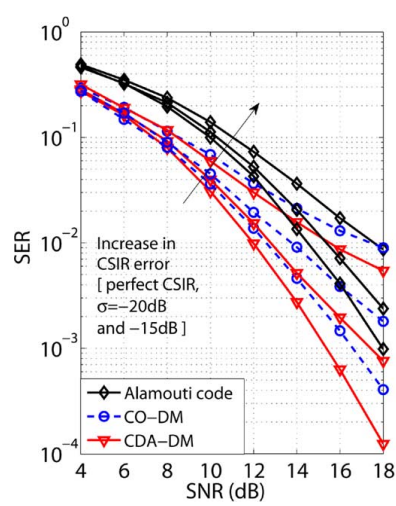

(b)

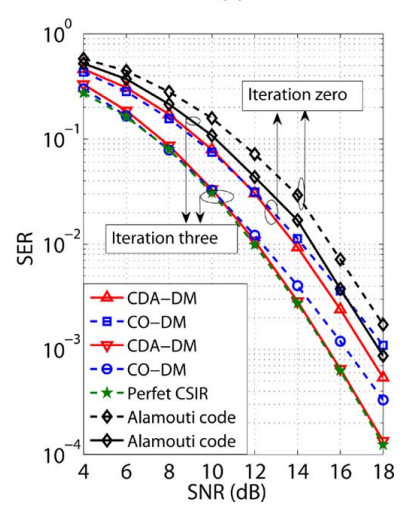

Fig. 2. Plot (a) gives the performance of Alamouti code, CDA-DM and CO-DM in CSTSK $(2,2,2,8)$, BPSK, under perturbed channel conditions with ML detection. Plot (b) gives the performance of Alamouti code, CDA-DM and CO-DM with semi-blind iterative detection/estimation algorithm at the receiver. (a) CSTSK $(2,2,2,8)$, BPSK, with perfect CSIR and CSIR error s of $-20 \mathrm{~dB}$ and $-15 \mathrm{~dB}$. (b) $\operatorname{CSTSK}(2,2,2,8)$, BPSK, with Semi-blind iterative detection.

CO-DM for SNRs higher than $10 \mathrm{~dB}$. Thus, CDA-DM offers a better coding gain than CO-DM without any capacity loss at medium and high SNRs.

\section{B. With Imperfect CSIR}

Fig. 2(a) illustrates the sensitivity of the SER performance under ML detection to CSIR perturbations, where $\sigma$ is the variance of the complex-valued circular symmetric Gaussian noise that models the channel estimation error. It is clear from Fig. 2(a) that the performance of the Alamouti code, the proposed and of the existing DMs degrades upon increasing the channel's estimation error variance. It is evident from the figure that the proposed CDA-DM perform significantly better than the existing CO-DM and the Alamouti code for all the estimation error variances considered. This is attributed to their higher coding gain.

Fig. 2(b) shows the SER performance of the Alamouti code, the CDA-DM against their CO-DM counterparts, with the aid of the detection/estimation algorithm based receiver proposed in [21]. It is evident from the plots that the proposed DMs give a better SER performance than the Alamouti code and the CO-DM for SNRs higher than $12 \mathrm{~dB}$. In $\operatorname{CSTSK}(2,2,2,8)$, BPSK, the proposed CDA-DMs have shown an SNR improvement of $1 \mathrm{~dB}$ at an SER of about $10^{-3}$ with respect to the CO-DMs. It is evident from Fig. 2(b) that the SER performance of CDA-DM at iteration three coincides with that of the perfect CSIR case and offers significant SNR gain with respect to Alamouti code.

\section{CONCLuSIONS}

A class of STBCs that falls into the STSK framework has been identified and termed as Decomposable Dispersion Codes. CDA codes were shown to belong to this class, which resulted in DMs with beneficial coding gains, which was achieved without compromising the achievable capacity. The proposed DMs have attained a better SER performance due to their higher coding gain under both perfect and imperfect CSIR conditions, as well as both with ML and with matched filtering based detectors.

\section{REFERENCES}

[1] R. Mesleh, H. Haas, C. Ahn, and S. Yun, "Spatial modulation-A new low complexity spectral efficiency enhancing technique," in Proc. First Int. Conf. Commun. Netw., Beijing, China, Oct. 2006, pp. 1-5.

[2] R. Mesleh, H. Haas, S. Sinanovic, C. Ahn, and S. Yun, "Spatial modulation," IEEE Trans. Veh. Technol., vol. 57, no. 4, pp. 2228-2242, 2008.

[3] M. Di Renzo and H. Haas, "Space shift keying (SSK-) MIMO over correlated rician fading channels: Performance analysis and a new method for transmit-diversity," IEEE Trans. Commun., vol. 59, no. 1, pp. 116-129, Jan. 2011.

[4] M. Di. Renzo, H. Haas, and P. M. Grant, "Spatial modulation for multiple-antenna wireless systems: A survey," IEEE Commun. Mag., vol. 49, no. 12 , pp. $182-191$, Dec. 2011.

[5] M. Di. Renzo and H. Haas, "Transmit-diversity for spatial modulation (SM): Towards the design of high-rate spatially-modulated space-time block codes," in IEEE Int. Conf. Communications, Kyoto, Japan, Jun. 5-9, 2011.

[6] S. Sugiura, S. Chen, and L. Hanzo, "A universal space-time architecture for multiple-antenna aided systems," IEEE Commun. Surveys Tuts., vol. 14, no. 2, pp. 401-420, 2012.

[7] R. Rajashekar and K. V. S. Hari, "Modulation diversity for spatial modulation using complex interleaved orthogonal design," in Proc. IEEE TENCON 2012, 2012, pp. 1-6.

[8] J. Jeganathan and A. Ghrayeb, "Space shift keying modulation for MIMO channels," IEEE Trans. Wireless Commun., vol. 8, no. 7, pp. 3692-3703, 2009.

[9] S. Sugiura, S. Chen, and L. Hanzo, "Coherent and differential space-time shift keying: A dispersion matrix approach," IEEE Trans. Commun., vol. 58, no. 11, pp. 3219-3230, 2010.

[10] D. Yang, C. Xu, L.-L. Yang, and L. Hanzo, "Transmit-diversity-assisted space-shift keying for colocated and distributed/cooperative MIMO elements," IEEE Trans. Veh. Technol., vol. 60, no. 6, pp. 2864-2869, Jul. 2011.

[11] L.-L. Yang, "Signal detection in antennahopping spacedivision multipleaccess systems with spaceshift keying modulation," IEEE Trans. Signal Process., vol. 60, no. 1, pp. 351-366, Jan. 2012.

[12] S. Sugiura, S. Chen, and L. Hanzo, "Generalized space-time shift keying designed for flexible diversity-, multiplexing- and complexitytradeoffs," IEEE Trans. Commun., vol. 10, no. 4, pp. 1144-1153, 2011.

[13] M. Di Renzo and H. Haas, "Performance comparison of different spatial modulation schemes in correlated fading channels," in Proc. IEEE Int. Conf. Commun., Cape Town, South Africa, May 2010.

[14] M. Di. Renzo and H. Haas, "Space shift keying (SSK) modulation: On the transmit-diversity/multiplexing trade-off," in Proc. IEEE Int. Conf. Commun. (ICC), Kyoto, 2011.

[15] E. Basar, U. Aygolu, E. Panayirci, and H. V. Poor, "Space-time block coding for spatial modulation," IEEE Trans. Commun., vol. 59, pp. 823-832, Mar. 2011.

[16] J. Jeganathan, A. Ghrayeb, and L. Szczecinski, "Spatial modulation: Optimal detection and performance analysis," IEEE Commun. Lett., vol. 12, no. 8, pp. 545-547, 2008.

[17] S. Sandhu, R. Nabar, D. Gore, and A. Paulraj, Introduction to SpaceTime Codes [Online]. Available: http://www.stanford.edu/group/sarg/ sandhu062503.pdf

[18] S. Sugiura, C. Xu, S. X. Ng, and L. Hanzo, "Reduced-complexity coherent versus non-coherent QAM-aided space-time shift keying," IEEE Trans. Commun., vol. 59, no. 11, pp. 3090-3101, Nov. 2011.

[19] B. A. Sethuraman and B. S. Rajan, "Full-divesity, high-rate space-time block codes from division algebras," IEEE Trans. Inf. Theory, vol. 49, no. 10, pp. 2596-2616, 2003.

[20] R. Rajashekar, K. V. S. Hari, and L. Hanzo, Structured Dispersion Matrices From Space-Time Block Codes for Space-Time Shift Keying Oct. 2012, arXiv: ID 0568130.

[21] S. Chen, S. Sugiura, and L. Hanzo, "Semi-blind joint channel estimation and data detection for space-time shift keying systems," IEEE Signal Process. Lett., vol. 17, no. 12, pp. 993-996, 2010. 